\section{OPEN ACCESS}

Edited by:

Udo S. Gaipl,

University Hospital Erlangen, Germany

Reviewed by:

Takaaki Tokito,

Kurume University, Japan

Jie $L i$,

Washington University in St. Louis,

United States

Yu Zhang,

Guizhou Provincial People's Hospital,

China

*Correspondence:

Zhen-Yu Ding

dingzhenyu@scu.edu.cn

${ }^{\text {t}}$ These authors have contributed equally to this work

Specialty section:

This article was submitted to

Cancer Immunity and Immunotherapy,

a section of the journal

Frontiers in Oncology

Received: 07 September 2020

Accepted: 11 May 2021

Published: 31 May 2021

Citation:

Fu Y, Zheng $Y$, Wang P-P and

Ding Z-Y (2021) Toxicities

of Immunotherapy for

Small Cell Lung Cancer.

Front. Oncol. 11:603658.

doi: 10.3389/fonc.2021.603658

\title{
Toxicities of Immunotherapy for Small Cell Lung Cancer
}

\author{
Yang $\mathrm{Fu}^{\dagger}$, Yue Zheng ${ }^{\dagger}$, Pei-Pei Wang and Zhen-Yu Ding * \\ Department of Biotherapy, Cancer Center, West China Hospital, West China Medical School, Sichuan University, Chengdu, China
}

Small cell lung cancer (SCLC), composing 15-20\% of lung cancer, is a fatal disease with extremely poor prognosis. In the past two decades, etoposide platinum doublet chemotherapy remained the only choice of therapy, with disappointing overall survival $\leq 1$ year for the metastatic disease. Novel treatments including immunotherapy are urgently needed and extensively explored. Recently, in two phase III trials, atezolizumab and durvalumab were shown to bring survival benefit to patients. While immunotherapy brings better outcome, it is accompanied by adverse events different from traditional treatments. Although these immune-related adverse events (irAEs) are generally mild and can be managed, some irAEs (myocarditis, pneumonitis) may be severe and even lifethreatening. Accompanying with the increasing application of immunotherapy in clinical practice, the irAEs should not be overlooked. In this review, the irAEs profile in clinical trials of immunotherapy for SCLC will be summarized, also its unique features compared with irAEs in other malignancies will be explored. This review may be helpful for the appropriate clinical use of immunotherapy for SCLC.

Keywords: small cell lung cancer, immune-related adverse events, neuromuscular toxicity, immune checkpoint inhibitors, death

\section{INTRODUCTION}

Small cell lung cancer (SCLC) is a fatal disease, with a 5-year survival less than $7 \%(1,2)$. Platinum doublet chemotherapy, usually combined with etopside, remains the standard-of-care for decades (3-5). Patients have a high initial response rates of $60 \%$, while most relapse within 6 months and decease within 10 months (5-7).

Immune checkpoint helps to maintain the immune stability, while during carcinogenesis it is hijacked by tumors to evade immune surveillance. Immune checkpoint inhibitors (ICIs) including antibodies against cytotoxic T-lymphocyte antigen 4 (CTLA-4) or programmed cell death 1 (PD-1)/ programmed cell death ligand 1 (PD-L1) act to reverse the immunosuppression imparted by tumor cells, either by blocking CTLA-4 pathway or interrupting the interaction between PD-1 and PD-L1 $(8,9)$. ICI has been widely used in a variety of malignancies, including non-small cell lung cancer, melanoma, triple-negative breast cancer, and non-Hodgkin lymphoma etc. $(8,10-12)$. Especially in SCLC which has a notorious reputation of poor prognosis, PD-L1 inhibitors including atezolizumab and durvalumab show promising efficacy $(9,13)$. 
While immunotherapy brings better outcome, it is accompanied by adverse events different from traditional treatments. Mounted immune response is directed to not only tumor, but also normal tissues and causes immune-related adverse events (irAEs) (14). Although these irAEs are generally mild and can be managed, some irAEs (myocarditis, pneumonitis) may be severe and even life-threatening $(15,16)$.

Recently, in two phase III trials (IMPower 133 and CASPIAN), atezolizumab and durvalumab were shown to bring survival benefit to patients $(9,13)$. Accompanying with the increasing application of immunotherapy in clinical practice, the irAEs should not be overlooked. In SCLC, due to the poor life expectance, also the high incidence of neurological complications, it is intriguing to ask whether the irAEs would be different from other tumors. This review provided a brief summary of irAEs from published clinical trials in the field of SCLC treatment.

\section{OVERVIEW OF SCLC}

SCLC is a distinct form of lung cancer, with dominant component of neuroendocrine tumor cells, and early and frequent distant metastases (17). Mutations in p53 gene (TP53) and retinoblastomal gene (RB1) are universal genetic events in SCLC (18). Studies also showed although SCLC harbors a high tumor mutational burden, tumor infiltrating lymphocytes are scarce in the microenvironment (19). Neither SCLC tends to express PD- L1, as it is found $\leq 20 \%$ tumor cells express PD-L1 (>1\%) (20, 21).

Etoposide plus platinum combination chemotherapy is recommended for metastatic SCLC patients (extensive stage, ES). For those in the limited stage (LS, non-metastatic), chest radiation at a dose of 45 Gy administered in 1.5 Gy fractions twice-daily for 30 days with chemotherapy, followed by prophylactic cranial irradiation is recommended (22). For relapsed or platinum-refractory SCLC, topotecan was the only approved drug by FDA in second-line treatment (23). Meanwhile, clinical trials on inhibitors of PARP, EZH2, WEE1, DLL3, and Aurora kinase etc. are all actively ongoing at this time, which is beyond the scope of this review (24). Here, we restrict our focus on the clinical data of the immunotherapy for SCLC.

\section{DATA ACQUISITION}

All relevant articles are identified by using the keywords "small cell lung cancer," "SCLC," "immunotherapy," "CTLA-4," "PD-1," "PD-L1," "clinical trial" on Pubmed, clinicaltrials.gov, Embase and Web of science. Abstracts and presentation were also reviewed from major conference including ASCO (https://www.asco.org/) and ELCC (https://www.esmo.org/) from 2015 to 2020.The literature or abstract was viewed, and those with only protocol design or lack of AEs results were excluded. Finally, fifteen studies involving ICIs for SCLC therapy with full description of the AEs were selected.

\section{LANDSCAPE OF IMMUNOTHERAPY FOR SCLC}

\section{First Line}

The first one being tested was ipilimumab, a fully human monoclonal antibody for CTLA-4. Following a successful phase II study (NCT00527735), a phase III trial (CA184-156) investigated the efficacy and safety of ipilimumab combined with chemotherapy $(25,26)$. However, the addition of ipilimumab failed to demonstrate any improvement in neither OS, ORR, nor duration of response. IMPower133 was a phase III trial to investigate the efficiency of atezolizumab (a humanized monoclonal PD-L1 antibody) combined with chemotherapy. The combination regimen showed benefit in both PFS $(5.2 \mathrm{~m}$ vs $4.7 \mathrm{~m}$ ) and OS (12.3 m vs $10.3 \mathrm{~m}$ ) (9). A similar good outcome was also achieved by durvalumab, another high-affinity human IgG1 monoclonal antibody for PD-L1. In the phase III CASPIAN study, the combination of durvalumab and chemotherapy achieved an OS of $13.3 \mathrm{~m}$ (13). The results of PD-1 antibodies seemed less favorably. KEYNOTE-604 was a phase III trial to investigate the efficacy of pembrolizumab (a humanized monoclonal IgG4 antibody) in ES-SCLC patients (27). The results showed that pembrolizumab significantly improves PFS, while OS narrowly had significant difference. Nivolumab is another monoclonal antibody for PD-1. A phase II randomized study (EA5161) evaluated the combination of nivolumab with EP for the ES-SCLC patients. Preliminary results were reported in ASCO 2020, nivolumab significantly improved PFS (5.5 and $4.7 \mathrm{~m}, \mathrm{p}=0.047$ ) in treated population while OS was no statistical difference $(11.3$ and $8.5 \mathrm{~m}, \mathrm{p}=$ 0.14) (28).

\section{Maintenance}

A phase III study (CHECKMATE-451) tested either nivolumab monotherapy, or nivolumab plus ipilimumab, or placebo as maintenance therapy after platinum-based first-line chemotherapy. However, nivolumab has a shorter OS compared with placebo (29). Another phase II, single-arm trial (NCT02359019) studied pembrolizumab as maintenance therapy. The 1-year PFS and OS rates were only 13 and $37 \%$ respectively (30).

\section{Second Line}

Salvage therapy for the relapsed SCLC is more difficult. At least two randomized controlled trial tested the efficacy of immunotherapy. In IFCT-1603 study, atezolizumab monotherapy was compared with topotecan or re-induction of initial chemotherapy (31). A phase III trial CHECKMATE-331 investigated the efficacy and safety of nivolumab monotherapy in the second line of therapy (32). Both trials demonstrated no superiority of immunotherapy over traditional chemotherapy.

Monotherapy seems inappropriate, and following studies tested combination therapy. In a multi-center, single arm, phase II study (NCT02551432), pembrolizumab was combined with chemotherapy drug paclitaxel (33). In another phase II study (NCT02484404), Durvalumab was tested in combination 
with olaparib (PARP inhibitors) (34). The preliminary reports of these small sample sized showed promising results.

\section{Third Line or Later}

Some early, small-scale studies were performed in these very latestaged patients. Nivolumab was the first ICIs approved by FDA for third-line therapy of SCLC, based on the results of CHECKMATE-032 in 2016 (35). KEYNOTE-028 (NCT02054806) and KEYNOTE-158 studies both tested pembrolizumab in the third line therapy. Based on the results, pembrolizumab monotherapy was approved to SCLC in thirdline or later $(36,37)$.

\section{TOXICITIES}

\section{Ipilimumab}

In the phase II study (NCT00527735), ipilimumab plus chemotherapy led to higher frequency of $\mathrm{AE}$, either any grade (49 and $43 \%$ ) or $\geq$ grade 3 (G3, 46 and 30\%) AE than chemotherapy alone. Common severe irAEs included G4 diarrhea $(n=1)$, G3 colitis $(n=1), G 4$ hepatitis $(n=2)$, and death $(n=1)$ attributed to hepatotoxicity (25). In the following phase III study (CA184-156), the combination also had higher incidence of irAEs of all grade (57\% in ipilimumab group, and $28 \%$ in control) or $\geq \mathrm{G} 3$ (20 and $2 \%$ ). Gastrointestinal and skin toxicity (34 and 29\%) were the most common irAEs in ipilimumab group. Endocrine irAEs occurred in $10 \%$ of patients in the ipilimumab group including hypothyroidism (3\%), hyperthyroidism (2\%), hypophysitis (1\%), and adrenal insufficiency $(1 \%)$. Two deaths due to colitis $(n=1)$ and ulcerative colitis $(n=1)$ were reported. The incidence of nervous system irAEs was $4 \%$ which involved $2 \%$ of peripheral sensory neuropathy (26).

\section{Atezolizumab}

In IMPower133 study, the incidence of AEs was 39.9\% in the atezolizumab group and $24.5 \%$ in the control group. The most common irAEs was rash (18.7\%), hypothyroidism (12.6\%), hepatitis $(7.1 \%)$, and hyperthyroidism $(5.6 \%)$. The less frequent $(\leq 5 \%)$ of irAEs were pneumonitis $(2.0 \%)$, colitis $(1.5 \%)$, rhabdomyolysis $(1.0 \%)$, severe cutaneous reaction $(1.0 \%)$, pancreatitis $(0.5 \%)$, nephritis $(0.5 \%)$, hypophysitis $(0.5 \%)$, and diabetes mellitus $(0.5 \%)$. Severe irAEs $(\geq \mathrm{G} 3)$ were rash $(2 \%)$, hepatitis $(1.5 \%)$, infusion-related reaction (2\%), and colitis (1\%) (9). In IFCT-1603 study, the incidence of AE, including $12.5 \%$ musculoskeletal or connective tissue disorders, $18.8 \%$ gastrointestinal disorders, $4.2 \%$ hepatitis, $4.2 \%$ colitis, $6.3 \%$ arthralgia, $2.1 \%$ hyperthyroidism and $2.1 \%$ hypothyroidism. No $\geq$ G3 irAE was reported (31).

\section{Durvalumab}

In CASPIAN study, three groups were enrolled, including durvalumab and chemotherapy, combo immunotherapy durvalumab and tremelimumab with chemotherapy, and chemotherapy. The incidences of $\geq \mathrm{G} 3$ AEs were 62.3, 70.3, and
$62.8 \%$ in each of these groups. G5 AEs were 4.9, 10.2 and 5.6\%, respectively. For G3-4 irAEs, the incidence was 5\% in durvalumab group and $\leq 1 \%$ in control group, and it was 20 and 3\% for any grade. Endocrine-related adverse events were the most common irAEs including hypothyroidism (9\%), hyperthyroidism $(5 \%)$, thyroiditis $(4 \%)$, type 1 diabetes mellitus (T1DM, $2 \%)$, rash $(2 \%)$, adrenal insufficiency $(<1 \%)$. The incidence of immune-related pneumonitis was $3 \%$ of all grades and $1 \%$ of G3-4. There were also reports of immunerelated colitis, pancreatic events, and hepatic events. Two immune-related deaths due to hepatotoxicity $(\mathrm{n}=1)$ and pneumonitis $(\mathrm{n}=1)$ were reported (13).

The phase II study (NCT02484404) was an exploratory study. In this study, nine patients (45\%) had G3-4 TRAEs including anemia, lymphopenia, thrombocytopenia, and hypophosphatemia. In five patients' hypothyroidism was observed attributed to immunotherapy (34).

\section{Pembrolizumab}

In the 1st line setting (KEYNOTE-604), when pembrolizumab used with chemotherapy, the incidence of irAEs (any grade) was $53 \%$, compared with $84 \%$ in the control group. Hypothyroidism (10.3\%), hyperthyroidism (6.7\%), and pneumonitis (4\%) were the most common. G3 irAEs occurred in only $7.2 \%$ of patients, and no G4-5 irAEs occurred (27). The only maintenance therapy study (NCT02359019) reported three categories of irAEs, rash $(\mathrm{n}=8)$, hypothyroidism $(\mathrm{n}=4)$, T1DM with diabetic ketoacidosis $(n=1)(30)$. In late lines of pembrolizumab monotherapy (KEYNOTE-028), the most frequent AEs were arthralgia, asthenia, and rash ( $\mathrm{n}=4$ each) as well as diarrhea and fatigue ( $\mathrm{n}=3$ each). Only two patients experienced G3 AE. One had G3 bilirubin elevation, and the other was a lethal case of colitis concurrent with G3 bilirubin elevation. Another similar study (KEYNOTE-158) reported AE of any grade and G3-5 were 33.7 and $5.1 \%$, respectively. Most common irAEs included hypothyroidism (12.1\%), hyperthyroidism (6.5\%), severe skin reactions (2.8\%), adrenal insufficiency, nephritis, pancreatitis, and pneumonitis (1.9\% each). G3 AE occurred in six patients, mostly manageable, and no fatal irAE was reported $(36,37)$. In an early-phase exploratory study (NCT02551432), AEs occurred in all patients. Pneumonia (19.2\%), T1DM (7.7\%), rush (7.7\%), and hypothyroidism (3.9\%) were among the most common irAEs. Four patients discontinued treatment (33).

\section{Nivolumab}

In the study CheckMate-331, TRAEs of all grade ( $\geq \mathrm{G} 3)$ occurred in $55 \%(14 \%)$ of nivolumab group, and $90 \%(73 \%)$ of chemotherapy group. There were five treatment-related death, two with nivolumab and three with chemotherapy. The incidences of irAEs (all grade) of endocrine, skin, gastrointestinal, liver, lung and kidney were $12,11,7,5,1$ and $<1 \%$ respectively (32). In study CheckMate-032, skin toxicity (any grade, 21.1\%) was the most common. Other irAEs including endocrine, gastrointestinal, hepatic, pulmonary and renal toxicity were $9.2,6.4,4.6,1.8$ and $0.9 \%$ respectively. The incidence of G3-4 pneumonitis, rash, aspartate aminotransferase increase was $1.8,0.9$, and $0.9 \%$, respectively. 
One immune-related encephalitis (grades 3-4) was reported. One death due to checkpoint inhibitor pneumonitis was noted (35). While in study CheckMate-451, the most frequently occurred serious AEs was pneumonitis (3.8\%). Other serious included colitis $(3.6 \%)$, endocrine $(2.5 \%)$, hepatitis $(0.7 \%)$, and nervous system (3.7\%). Myocarditis was reported in two cases $(0.7 \%)$ in group. AEs in nervous system were encephalitis $(n=2)$, myasthenia gravis $(n=1)$, and Guillain-Barré syndrome $(n=1)$. There were eight treatment-related deaths in the nivolumab group versus one in the control group (29). In study EA5161, the incidence of grade $3 / 4$ TRAEs was $77 \%$ vs $62 \%$. Treatmentrelated fatal adverse events were similar in the two groups $(n=9$ and 7) (28).

\section{DISCUSSION}

This review summarized 15 trials in SCLC immunotherapy, including phase III $(\mathrm{n}=5)$ and phase I/II trials $(\mathrm{n}=10$, Figure 1). Among them, IMpower133, CASPIAN, CA184-156, KEYNOTE-604 and EA5161 evaluated the efficacy of atezolizumab, durvalumab, ipilimumab, pembrolizumab, or nivolumab, when combined with chemotherapy. CheckMate331 and IFCT-1603 tested the efficacy of nivolumab and atezolizumab monotherapy in 2nd-line. Six trials investigated efficacy and safety of ICIs in later-line or maintenance treatment. Most trials were performed in ICIs combined with chemotherapy. More studies are ongoing (Table 1).

When all the 15 trials combined for analysis, PD-1/PD- L1 inhibitors had a better tolerance than CTLA-4 inhibitors (Figure 2A). Dermal events (23.8\%), colitis (5.6\%), hepatitis (4.3\%), hypophysitis $(0.4 \%)$, myasthenic $(0.3 \%)$, and myocarditis $(0.3 \%)$ were more common with CTLA-4 inhibitors, whereas

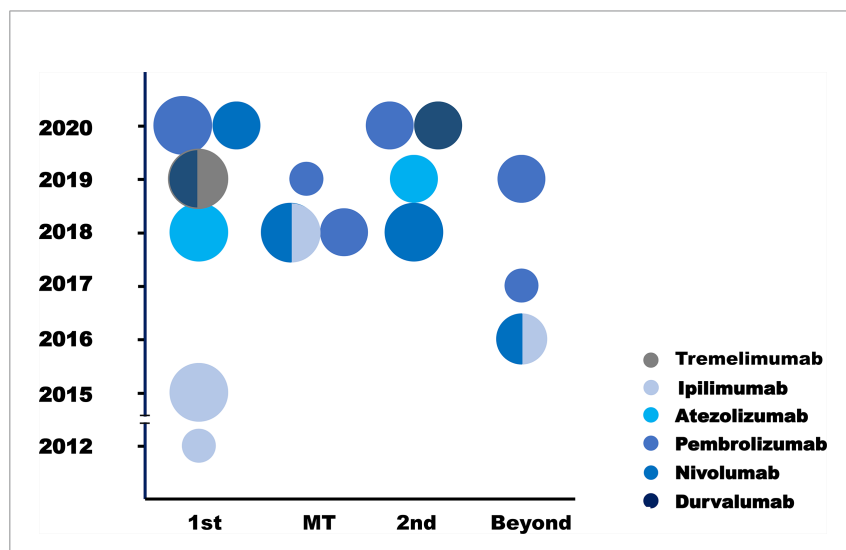

FIGURE 1 | Clinical trials of ICls in SCLC. Each circle standed for one trial. The different circle areas represented phase I, phase II and phase III trials respectively, and different color meant different drugs. $Y$ axis stood for the year of the publication of each trial, and $X$ trials was the clinical situation where each trial was performed. 1st, first line; MT, maintenance therapy; 2nd, second line; Beyond, later line. pneumonitis $(3.7 \%)$, thyroid events $(14.3 \%)$, pancreatic events (1.0\%), and rheumatic events $(0.2 \%)$ were more common with PD-1/PD- L1 inhibitors. It was also interesting to observe the difference of toxicities between PD-1 and PD-L1 inhibitors. Generally, the rate of irAE by PD-L1 inhibitors was lower than that of PD-1 inhibitors, including pneumonitis (4.3\% vs $2.1 \%$ ), dermal events ( $12.4 \%$ vs $8.1 \%)$, colitis $(2.3 \%$ vs $1.7 \%)$, adrenal insufficiency $(0.7 \%$ vs $0.2 \%)$, nephritis $(0.6 \%$ vs $0.2 \%)$, myositis $(0.4 \%$ vs 0$)$, rheumatic disease $(0.4 \%$ vs 0$)$, hypophysitis $(0.2 \%$ vs 0 ), and myocarditis ( $0.1 \%$ vs 0 , Figure $2 \mathbf{B})$.

In CheckMate-451 trial, the frequency of irAEs of the nivolumab plus ipilimumab group was higher than that of nivolumab group. Not only occurrence, but the severity (frequency of $\geq \mathrm{G} 3$ irAEs) was also worse in the combo therapy. Similarly, immunotherapy plus chemotherapy showed better efficacy in IMpower133 and CASPIAN study, but at the price of more irAE events. Furthermore, adding ipilimumab to this combination brought no additional benefit, but significantly higher toxicities.

The exact pathophysiology of irAEs is unclear, but the toxicity between CTLA-4 and PD-L1/PD-1 inhibitors is quite different. Pituitary cells translocate to express CTLA-4. The CTLA-4 antibody binds to the pituitary and induces lymphocyte infiltration, and tissue destruction is triggered $(38,39)$. PD-L1 was highly expressed on the surface of myocardial cells in two patients with immune myocarditis, leading to the recognition of myocardial and tumor surface antigens by the same $\mathrm{T}$ cell clone, which ultimately cause destruction of organ (16). In Keynote001 trial, 10 patients were newly diagnosed with hypothyroidism after receiving pembrolizumab, and eight of them were diagnosed with anti-thyroid antibody (40). It was suggested that irAE may be associated with autoantibodies. CTLA-4 Inhibitors reduce the number and activity of Treg cells, resulting in increased activity of TH17 cells and increased IL17 release, contributing to the onset of immune-related colitis (41-43).

Because SCLC is a kind of neuroendocrine tumor, also autoimmune encephalitis was frequently reported for this disease, we proposed there might be an increased occurrence of neuromuscular toxicity during the immunotherapy. To test this hypothesis, we performed a pooled analysis of the reported neuromuscular toxicity from the above trials. We found less occurrence in the control group, compared with that in immunotherapy group (Figure 3A). To confirm this observation, we performed a similar analysis in NSCLC trials. Conversely, immunotherapy and control groups had comparable toxicity (Figure 3B). This further supported the notion the neuromuscular toxicity of immunotherapy was specifically restricted in SCLC.

We paid special attention to the fatal toxicities. Immunotherapy and chemotherapy had a similar incidence of treatment-related death for SCLC patients. Totally 36 and 27 death events occurred from seven head-to-head trials respectively (Figure 4). From all the trials, the most common reason of reported death were sepsis $(n=7)$ and pneumonitis $(n=7)$, followed by multiorgan failure $(n=3)$, hematologic 


\begin{tabular}{|c|c|c|c|c|c|c|c|c|}
\hline Trial & & Treatment & Phase & Intervention & Population & Patients & Therapy & Status \\
\hline NCT02580994 & REACTION & First line & ॥ & RCT & ES-SCLC & 125 & Pembrolizumab + EC/EP vs EC/EP & Recruiting \\
\hline NCT02402920 & & First line & 1 & Parallel & SCLC & 80 & Pembrolizumab + Concurrent Chemo/Radiotherapy & Recruiting \\
\hline NCT02963090 & & Second line & $\|$ & $\mathrm{RCT}$ & Relapsed & 98 & Pembrolizumab vs Topotecan & Active \\
\hline NCT03371979 & & Second line & I/II & Single arm & Relapsed & 84 & Pembrolizumab + Pegzilarginase(AEB1102) & Active \\
\hline NCT04358237 & LUPER & Second line & $\mid / / 1$ & Single arm & Relapsed & 42 & Pembrolizumab + Lurbinectedin (PM01183) & Not yet recruiting \\
\hline NCT03253068 & & Second line & $\|$ & Single arm & Relapsed & 25 & Pembrolizumab + Amurubicin & Recruiting \\
\hline NCT04173325 & & Second line & 1 & Single arm & Relapsed & 10 & Nivolumab + Irinotecan & Recruiting \\
\hline NCT03406715 & & Second line & $\|$ & Multicohort & SCLC & 40 & Nivolumab+ Ipilimumab+ Dendritic Cell p53 Vac & Recruiting \\
\hline NCT03083691 & BIOLUMA & Second line & ॥ & Multicohort & Relapsed & 106 & Nivolumab + Ipilimumab & Recruiting \\
\hline NCT03670056 & & Second line & $\|$ & Single arm & Relapsed & 40 & Nivolumab + Ipilimumab & Recruiting \\
\hline NCT03728361 & & Second line & ॥ & Multicohort & Relapsed & 53 & Nivolumab + Temozolomide & Recruiting \\
\hline NCT03575793 & & Second line & |/II & Parallel & Relapsed & 55 & Nivolumab + Ipilimumab + Plinabulin & Recruiting \\
\hline NCT03662074 & & Second line & $\|$ & Single arm & Relapsed & 14 & Nivolumab + Gemcitabine & Active \\
\hline NCT02247349 & & Second line & $\mid / / 1$ & Parallel & Relapsed & 172 & BMS-986012 + Nivolumab vs BMS-986012 & Active \\
\hline NCT03325816 & & Maintenance & |//I & Single arm & ES-SCLC & 9 & Nivolumab + Lutathera & Active \\
\hline NCT03958045 & & Maintenance & $\|$ & Single arm & SCLC & 36 & Nivolumab + Rucaparib & Recruiting \\
\hline NCT02046733 & STIMULI & Maintenance & $\|$ & Parallel & LS-SCLC & 264 & Nivolumab + Ipilimumab After Chemo-radiotherapy & Active \\
\hline NCT04189094 & & First line & ॥ & $\mathrm{RCT}$ & LS-SCLC & 140 & Sintilimab + EC/EP + RT vs EC/EP + RT & Not yet recruiting \\
\hline NCT04192682 & & Second line & $\|/\| \|$ & Single arm & Relapsed & 40 & Sintilimab + Anlotinib after Chemo-radiotherapy & Not yet recruiting \\
\hline NCT04055792 & & Beyond & $\|$ & $\mathrm{RCT}$ & ES-SCLC & 52 & Sintilimab + Anlotinib vs Anlotinib & Recruiting \\
\hline NCT03983759 & & Maintenance & $\|$ & Single arm & ES-SCLC & 40 & Sintilimab After Chemotherapy + R-CIK & Recruiting \\
\hline NCT04449861 & ORIENTAL & First line & IIIlb & Single arm & ES-SCLC & 300 & Durvalumab + EC/EP & Not yet recruiting \\
\hline NCT03509012 & CLOVER & First line & 1 & Multicohort & SCLC & 360 & Durvalumab \pm Tremelimumab + EC/EP + Radiotherapy & Active \\
\hline NCT04361825 & & Second line & $\|$ & Single arm & Relapsed & 45 & Durvalumab + AZD6738 & Recruiting \\
\hline NCT02701400 & & Second line & $\|$ & Parallel & Relapsed & 18 & Durvalumab + Tremelimumab \pm RT & Active \\
\hline NCT02937818 & & Second line & $\|$ & Parallel & Refractory $^{*}$ & 72 & Durvalumab + Tremelimumab vs AZD1775 + carboplatin vs AZD6738 + Olaparib & Active \\
\hline NCT04314297 & & Maintenance & $\|$ & Single arm & ES-SCLC & 33 & Durvalumab + Anlotinib after Chemo-radiotherapy & Not yet recruiting \\
\hline NCT04472949 & & Maintenance & $\|$ & Single arm & ES-SCLC & 46 & RT+ Durvalumab after Durvalumab + EC & Not yet recruiting \\
\hline NCT03585998 & & Maintenance & $\|$ & Single arm & LS-SCLC & 51 & Durvalumab after Chemo-radiotherapy + Durvalumab & Active \\
\hline NCT03703297 & ADRIATIC & Maintenance & III & $\mathrm{RCT}$ & LS-SCLC & 600 & $\begin{array}{l}\text { 4Durvalumab + 4Placebo;Durvalumab } \\
\text { 4Durvalumab + 4Tremelimumab;:Durvalumab } \\
4 \text { Placebo; Placebo }\end{array}$ & Recruiting \\
\hline NCT03923270 & & Maintenance & I & Parallel & ES-SCLC & 54 & RT followed by Durvalumab or Durvalumab + Tremelimumab or Olaparib & Recruiting \\
\hline NCT04256421 & SKYSCRAPER-02 & First line & III & $\mathrm{RCT}$ & ES-SCLC & 400 & Atezolizumab + EC+ Tiragolumab vs Atezolizumab + EC & Recruiting \\
\hline NCT03041311 & & First line & ॥ & $\mathrm{RCT}$ & ES-SCLC & 105 & Atezolizumab + EC + Trilaciclib(G1T28) vs Atezolizumab + EC & Active \\
\hline NCT03540420 & & First line & $\|$ & RCT & LS-SCLC & 212 & Atezolizumab vs standard care after Chemo-radiotherapy & Recruiting \\
\hline NCT04028050 & MAURIS & First line & IIllb & $\mathrm{RCT}$ & ES-SCLC & 150 & Atezolizumab + EC & Recruiting \\
\hline NCT04422210 & & First line & $\mathrm{lb}$ & Single arm & ES-SCLC & 62 & Venetoclax + Atezolizumab + EC & Recruiting \\
\hline NCT03262454 & & Second line & $\|$ & Single arm & Relapsed & 35 & Radiotherapy Followed by Atezolizumab & Recruiting \\
\hline NCT03059667 & & Second line & $\|$ & $\mathrm{RCT}$ & SCLC & 70 & Atezolizumab vs Topotecan/Etoposide/Carboplatin & Active \\
\hline NCT04402788 & RAPTOR & Second line & $\|/\| \|$ & $\mathrm{RCT}$ & ES-SCLC & 324 & Atezolizumab + RT vs Atezolizumab & Not yet recruiting \\
\hline NCT04308785 & & Maintenance & $\|$ & RCT & LS-SCLC & 242 & Atezolizumab + EC/EP+ radiotherapy vs EC/EP + radiotherapy & Not yet recruiting \\
\hline NCT04462276 & TREASURE & Maintenance & ॥ & $\mathrm{RCT}$ & ES-SCLC & 104 & Atezolizumab + RT vs Atezolizumab after Atezolizumab + EC & Not yet recruiting \\
\hline NCT04373369 & & Maintenance & $\|$ & Single arm & ES-SCLC & 33 & Atezolizumab + Vorolanib & Not yet recruiting \\
\hline NCT03811002 & & First line & $\|/\| 1 \|$ & $\mathrm{RCT}$ & LS-SCLC & 506 & Atezolizumab + EC/EP + RT vs EC/EP + RT & Recruiting \\
\hline NCT04418648 & & consolidation & $\|$ & $\mathrm{RCT}$ & LS-SCLC & 170 & Toripalimab vs Observation & Not yet recruiting \\
\hline NCT04363255 & & Maintenance & $\|$ & Single arm & ES-SCLC & 20 & EC/EP followed by Toripalimab + Anlotinib & Not yet recruiting \\
\hline NCT04012606 & & First line & III & RCT & ES-SCLC & 420 & Toripalimab(JS001) + EC/EP vs EC/EP & Recruiting \\
\hline
\end{tabular}


A

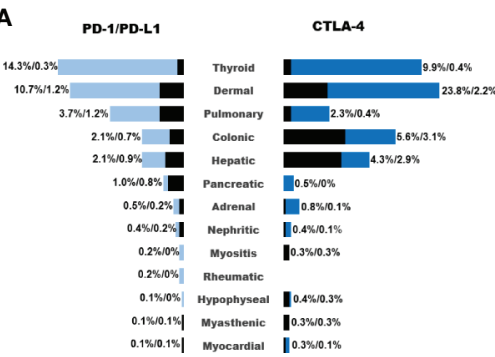

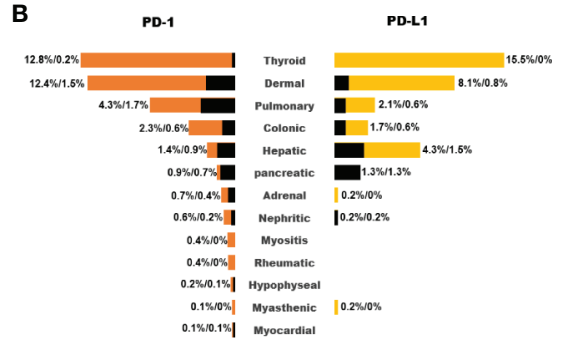

FIGURE 2 | List of common irAEs for different ICls (A: PD-1/PD-L1 inhibitors vs CTLA-4 inhibitors; B: PD-1 inhibitors vs PD-L1 inhibitors). Colored and black bar indicated the occurrence of irAEs of any grade and $\geq$ grade 3 .

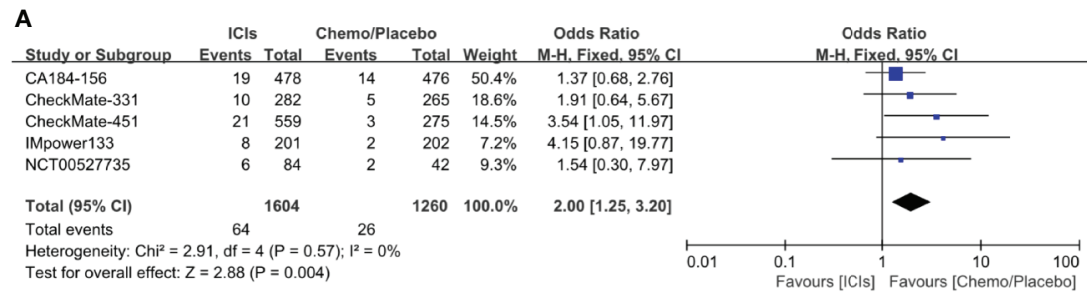

B

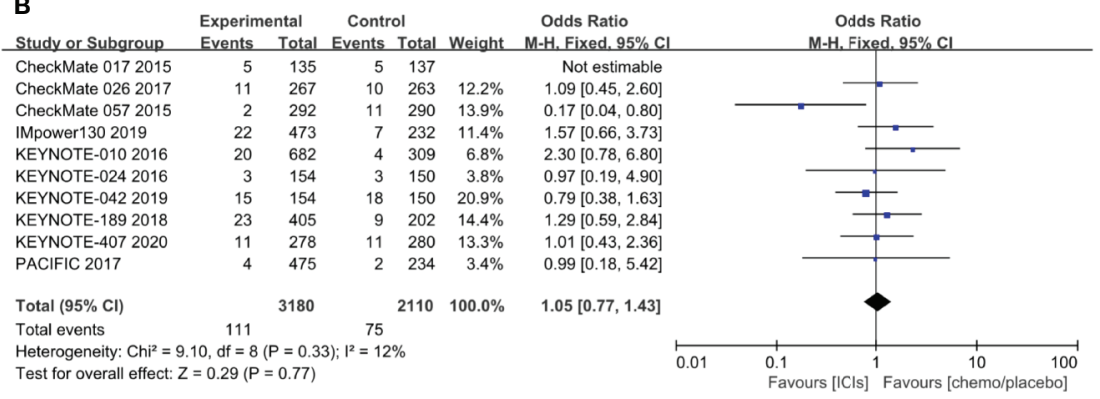

FIGURE 3 | Pooled analysis of neuromuscular toxicity in SCLC (A) and NSCLC (B).

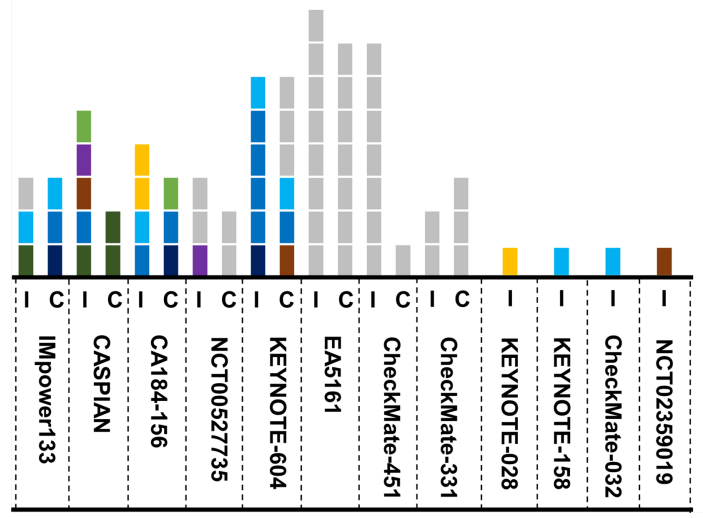

Multiorgan failure

Hepatic

Sepsis

Pneumonic

Hematologic

Cardiotoxicity

Gastroenteric

Not otherwise specified

Unreported

C, chemo/placebo group.
C 
disease $(n=2)$, cardiotoxicity $(n=3)$, hepatitis $(n=3)$, and other unspecified cause $(n=2)$.

\section{CONCLUSION}

This paper reviewed the current status of immunotherapy in SCLC. Immunotherapy brings new hope to this formidable disease, and also unprecedented toxicity profile. Immunotherapy combined with either chemotherapy or other immunotherapies, led to higher occurrence of AE than immunotherapy alone. The toxicity of immunotherapy in SCLC seemed to be different with

\section{REFERENCES}

1. Byers LA, Rudin CM. Small Cell Lung Cancer: Where Do We Go From Here? Cancer (2015) 121(5):664-72. doi: 10.1002/cncr.29098

2. Gaspar LE, McNamara EJ, Gay EG, Putnam JB, Crawford J, Herbst RS, et al. Small-Cell Lung Cancer: Prognostic Factors and Changing Treatment Over 15 Years. Clin Lung Cancer (2012) 13(2):115-22. doi: 10.1016/ j.cllc.2011.05.008

3. Socinski MA, Smit EF, Lorigan P, Konduri K, Reck M, Szczesna A, et al. Phase III Study of Pemetrexed Plus Carboplatin Compared With Etoposide Plus Carboplatin in Chemotherapy-Naive Patients With Extensive-Stage SmallCell Lung Cancer. J Clin Oncol (2009) 27(28):4787-92. doi: 10.1200/ JCO.2009.23.1548

4. Früh M, De Ruysscher D, Popat S, Crinò L, Peters S, Felip E, Esmo Guidelines Working Group. Small-Cell Lung Cancer (SCLC): ESMO Clinical Practice Guidelines for Diagnosis, Treatment and Follow-Up. Ann Oncol (2013) 24 Suppl 6:vi99-105. doi: 10.1093/annonc/mdt178

5. Farago Anna F, Keane Florence K. Current Standards for Clinical Management of Small Cell Lung Cancer. Transl Lung Cancer Res (2018) 7:69-79. doi: 10.21037/tlcr.2018.01.16

6. Jones GS, Elimian K, Baldwin DR, Hubbard RB, McKeever TM. A Systematic Review of Survival Following Anti-Cancer Treatment for Small Cell Lung Cancer. Lung Cancer (2020) 141:44-55. doi: 10.1016/j.lungcan.2019.12.015

7. Demedts IK, Vermaelen KY, van Meerbeeck JP. Treatment of Extensive-Stage Small Cell Lung Carcinoma: Current Status and Future Prospects. Eur Respir J (2010) 35:202-15. doi: 10.1183/09031936.00105009

8. Mok TSK, Wu Y-L, Kudaba I, Kowalski DM, Cho BC, Turna HZ, et al. Pembrolizumab Versus Chemotherapy for Previously Untreated, PD-L1expressing, Locally Advanced or Metastatic Non-Small-Cell Lung Cancer (KEYNOTE-042): A Randomised, Open-Label, Controlled, Phase 3 Trial. Lancet (2019) 393:1819-30. doi: 10.1016/S0140-6736(18)32409-7

9. Horn L, Mansfield AS, Szczęsna A, Havel L, Krzakowski M, Hochmair MJ, et al. First-Line Atezolizumab Plus Chemotherapy in Extensive-Stage SmallCell Lung Cancer. N Engl J Med (2018) 379:2220-9. doi: 10.1056/ NEJMoa1809064

10. Gandhi L, Rodríguez-Abreu D, Gadgeel S, Esteban E, Felip E, De Angelis F, et al. Pembrolizumab Plus Chemotherapy in Metastatic Non-Small-Cell Lung Cancer. N Engl J Med (2018) 378:2078-92. doi: 10.1056/NEJMoa1801005

11. Schmid P, Adams S, Rugo HS, Schneeweiss A, Barrios $\mathrm{CH}$, Iwata $\mathrm{H}$, et al. Atezolizumab and Nab-Paclitaxel in Advanced Triple-Negative Breast Cancer. N Engl J Med (2018) 379:2108-21. doi: 10.1056/NEJMoa1809615

12. Ansell SM, Lesokhin AM, Borrello I, Halwani A, Scott EC, Gutierrez M, et al. PD-1 Blockade With Nivolumab in Relapsed or Refractory Hodgkin's Lymphoma. N Engl J Med (2015) 372:311-9. doi: 10.1056/NEJMoa1411087

13. Paz-Ares L, Dvorkin M, Chen Y, Reinmuth N, Hotta K, Trukhin D, et al. Durvalumab Plus Platinum-Etoposide Versus Platinum-Etoposide in FirstLine Treatment of Extensive-Stage Small-Cell Lung Cancer (CASPIAN): A Randomised, Controlled, Open-Label, Phase 3 Trial. Lancet (2019) 394:192939. doi: 10.1016/S0140-6736(19)32222-6

14. Postow MA, Sidlow R, Hellmann MD. Immune-Related Adverse Events Associated With Immune Checkpoint Blockade. N Engl J Med (2018) 378:158-68. doi: 10.1056/NEJMra1703481 those in NSCLC, esp. for neuromuscular toxicity. This review may be helpful for the appropriate clinical use of immunotherapy for SCLC.

\section{AUTHOR CONTRIBUTIONS}

Z-YD and YZ contributed conception. YF drafted the manuscript, Z-YD reviewed the manuscript, and P-PW edited the manuscript. All authors contributed to the article and approved the submitted version.

15. Hu Y-B, Zhang Q, Li H-J, Michot JM, Liu H-B, Zhan P, et al. Evaluation of Rare But Severe Immune Related Adverse Effects in PD-1 and PD-L1 Inhibitors in Non-Small Cell Lung Cancer: A Meta-Analysis. Transl Lung Cancer Res (2017) 6:S8-20. doi: 10.21037/tlcr.2017.12.10

16. Johnson DB, Balko JM, Compton ML, Chalkias S, Gorham J, Xu Y, et al. Fulminant Myocarditis With Combination Immune Checkpoint Blockade. N Engl J Med (2016) 375:1749-55. doi: 10.1056/NEJMoa1609214

17. Gazdar AF, Bunn PA, Minna JD. Small-Cell Lung Cancer: What We Know, What We Need to Know and the Path Forward. Nat Rev Cancer (2017) 17 (12):725-37. doi: 10.1038/nrc.2017.87

18. George J, Lim JS, Jang SJ, Cun Y, Ozretić L, Kong G, et al. Comprehensive Genomic Profiles of Small Cell Lung Cancer. Nature (2015) 524:47-53. doi: $10.1038 /$ nature 14664

19. Iams WT, Shiuan E, Meador CB, Roth M, Bordeaux J, Vaupel C, et al. Improved Prognosis and Increased Tumor-Infiltrating Lymphocytes in Patients Who Have Sclc With Neurologic Paraneoplastic Syndromes. J Thorac Oncol (2019) 14:1970-81. doi: 10.1016/j.jtho.2019.05.042

20. Yu H, Batenchuk C, Badzio A, Boyle TA, Czapiewski P, Chan DC, et al. Pd-L1 Expression by Two Complementary Diagnostic Assays and Mrna In Situ Hybridization in Small Cell Lung Cancer. J Thorac Oncol (2017) 12:110-20. doi: 10.1016/j.jtho.2016.09.002

21. Zhao X, Kallakury B, Chahine JJ, Hartmann D, Zhang Y, Chen Y, et al. Surgical Resection of SCLC: Prognostic Factors and the Tumor Microenvironment. J Thorac Oncol (2019) 14:914-23. doi: 10.1016/j.jtho.2019.01.019

22. Turrisi AT, Kim K, Blum R, Sause WT, Livingston RB, Komaki R, et al. Twice-Daily Compared With Once-Daily Thoracic Radiotherapy in Limited Small-Cell Lung Cancer Treated Concurrently With Cisplatin and Etoposide. N Engl J Med (1999) 340:265-71. doi: 10.1056/NEJM199901283400403

23. Ardizzoni A, Hansen H, Dombernowsky P, Gamucci T, Kaplan S, Postmus P, et al. Topotecan, a New Active Drug in the Second-Line Treatment of SmallCell Lung Cancer: A Phase II Study in Patients With Refractory and Sensitive Disease. The European Organization for Research and Treatment of Cancer Early Clinical Studies Group and New Drug Development Office, and the Lung Cancer Cooperative Group. J Clin Oncol (1997) 15:2090-6. doi: 10.1200/ JCO.1997.15.5.2090

24. Sabari Joshua K, Lok Benjamin H, Laird James H, Poirier John T, Rudin Charles M. Unravelling the Biology of SCLC: Implications for Therapy. Nat Rev Clin Oncol (2017) 14:549-61. doi: 10.1038/nrclinonc.2017.71

25. Reck M, Bondarenko I, Luft A, Serwatowski P, Barlesi F, Chacko R, et al. Ipilimumab in Combination With Paclitaxel and Carboplatin as First-Line Therapy in Extensive-Disease-Small-Cell Lung Cancer: Results From a Randomized, Double-Blind, Multicenter Phase 2 Trial. Ann Oncol (2013) 24:75-83. doi: 10.1093/annonc/mds213

26. Reck M, Luft A, Szczesna A, Havel L, Kim S-W, Akerley W, et al. Phase III Randomized Trial of Ipilimumab Plus Etoposide and Platinum Versus Placebo Plus Etoposide and Platinum in Extensive-Stage Small-Cell Lung Cancer. J Clin Oncol (2016) 34:3740-8. doi: 10.1200/JCO.2016.67.6601

27. Rudin CM, Awad MM, Navarro A, Gottfried M, Peters S, Csőszi T, et al. Pembrolizumab or Placebo Plus Etoposide and Platinum as First-Line Therapy for Extensive-Stage Small-Cell Lung Cancer: Randomized, DoubleBlind, Phase Iii KEYNOTE-604 Study. J Clin Oncol (2020) 38:2369-79. doi: $10.1200 /$ JCO.20.00793 
28. Leal TA, Wang Y, Dowlati A, Lewis DA, Chen Y, Mohindra AR, et al. Randomized Phase II Clinical Trial of Cisplatin/Carboplatin and Etoposide (CE) Alone or in Combination With Nivolumab as Frontline Therapy for Extensive-Stage Small Cell Lung Cancer (ES-SCLC): ECOG-ACRIN EA5161. J Clin Oncol (2020) 38(no. 15_suppl):9000. doi: 10.1200/JCO.2020.38. 15_suppl.9000

29. Owonikoko TK, Kim HR, Govindan R, Ready N, Reck M, Peters S, et al. Nivolumab Plus Ipilimumab, Nivolumab, or Placebo as Maintenance Therapy in Patients With Extensive Disease Small Cell Lung Cancer After First-Line Platinum-Based Chemotherapy: Results From the Double-Blind, Randomized Phase 3 CheckMate 451 Study. Ann Oncol (2019) 30(suppl_2):ii77-80. doi: 10.1093/annonc/mdz094

30. Gadgeel SM, Pennell NA, Fidler MJ, Halmos B, Bonomi P, Stevenson J, et al. Phase II Study of Maintenance Pembrolizumab in Patients With ExtensiveStage Small Cell Lung Cancer (SCLC). J Thorac Oncol (2018) 13:1393-9. doi: $10.1016 /$ j.jtho.2018.05.002

31. Pujol J-L, Greillier L, Audigier-Valette C, Moro-Sibilot D, Uwer L, Hureaux J, et al. A Randomized non-Comparative Phase Ii Study of Anti-Programmed Cell Death-Ligand 1 Atezolizumab or Chemotherapy as Second-Line Therapy in Patients With Small Cell Lung Cancer: Results From the IFCT-1603 Trial. J Thorac Oncol (2019) 14:903-13. doi: 10.1016/j.jtho.2019.01.008

32. Spigel DR, Vicente D, Ciuleanu TE, Gettinger S, Peters S, Horn L, et al. Second-Line Nivolumab in Relapsed Small-Cell Lung Cancer: CheckMate 331. Ann Oncol (2021) 32(5):631-41. doi: 10.1016/j.annonc.2021.01.071

33. Kim Y-J, Keam B, Ock C-Y, Song S, Kim M, Kim SH, et al. A Phase II Study of Pembrolizumab and Paclitaxel in Patients With Relapsed or Refractory Small-Cell Lung Cancer. Lung Cancer (2019) 136:122-8. doi: 10.1016/j.lungcan.2019.08.031

34. Thomas A, Vilimas R, Trindade C, Erwin-Cohen R, Roper N, Xi L, et al. Durvalumab in Combination With Olaparib in Patients With Relapsed SCLC: Results From a Phase II Study. J Thorac Oncol (2019) 14:1447-57. doi: 10.1016/j.jtho.2019.04.026

35. Antonia SJ, López-Martin JA, Bendell J, Ott PA, Taylor M, Eder JP, et al. Nivolumab Alone and Nivolumab Plus Ipilimumab in Recurrent Small-Cell Lung Cancer (CheckMate 032): A Multicentre, Open-Label, Phase 1/2 Trial. Lancet Oncol (2016) 17:883-95. doi: 10.1016/S1470-2045(16)30098-5

36. Ott PA, Elez E, Sandrine H, Kim D-W, Morosky A, Saraf S, et al. Pembrolizumab in Patients With Extensive-Stage Small-Cell Lung Cancer: Results From the Phase Ib KEYNOTE-028 Study. J Clin Oncol (2017) 35:3823-9. doi: 10.1200/JCO.2017.72.5069
37. Chung HC, Piha-Paul SA, Lopez-Martin J, Schellens JHM, Kao S, Miller WH, et al. Pembrolizumab After Two or More Lines of Previous Therapy in Patients With Recurrent or Metastatic Sclc: Results From the KEYNOTE-028 and KEYNOTE-158 Studies. J Thorac Oncol (2020) 15(4):618-27. doi: 10.1016/j.jtho.2019.12.109

38. Iwama S, De Remigis A, Callahan MK, Slovin SF, Wolchok JD, Caturegli P, et al. Pituitary Expression of CTLA-4 Mediates Hypophysitis Secondary to Administration of CTLA-4 Blocking Antibody. Sci Transl Med (2014) 6:230ra45. doi: 10.1126/scitranslmed.3008002

39. Caturegli P, Di Dalmazi G, Lombardi M, Grosso F, Larman HB, Larman T, et al. Hypophysitis Secondary to Cytotoxic T-Lymphocyte-Associated Protein 4 Blockade: Insights Into Pathogenesis From an Autopsy Series. Am J Pathol (2016) 186:3225-35. doi: 10.1016/j.ajpath.2016.08.020

40. Osorio JC, Ni A, Chaft JE, Pollina R, Kasler MK, Stephens D, et al. AntibodyMediated Thyroid Dysfunction During T-cell Checkpoint Blockade in Patients With Non-Small-Cell Lung Cancer. Ann Oncol (2017) 28:583-9. doi: 10.1093/annonc/mdw640

41. von Euw E, Chodon T, Attar N, Jalil J, Koya RC, Comin-Anduix B, et al. CTLA4 Blockade Increases Th17 Cells in Patients With Metastatic Melanoma. J Transl Med (2009) 7:35. doi: 10.1186/1479-5876-7-35

42. Anderson R, Theron AJ, Rapoport BL. Immunopathogenesis of Immune Checkpoint Inhibitor-Related Adverse Events: Roles of the Intestinal Microbiome and Th17 Cells. Front Immunol (2019) 10:2254. doi: 10.3389/ fimmu.2019.02254

43. Esfahani K, Miller WH. Reversal of Autoimmune Toxicity and Loss of Tumor Response by Interleukin-17 Blockade. N Engl J Med (2017) 376:1989-91. doi: 10.1056/NEJMc1703047

Conflict of Interest: The authors declare that the research was conducted in the absence of any commercial or financial relationships that could be construed as a potential conflict of interest.

Copyright (c) $2021 \mathrm{Fu}$, Zheng, Wang and Ding. This is an open-access article distributed under the terms of the Creative Commons Attribution License (CC BY). The use, distribution or reproduction in other forums is permitted, provided the original author(s) and the copyright owner(s) are credited and that the original publication in this journal is cited, in accordance with accepted academic practice. No use, distribution or reproduction is permitted which does not comply with these terms. 